\title{
Brain Ciliary Neurotrophic Factor (CNTF) and hypothalamic control of energy homeostasis
}

\author{
Claire-Marie VACHER \\ Odile COUVREUR \\ Elsa BASIRE \\ Alain AUBOURG \\ Delphine CRÉPIN \\ Flavien BERTHOU \\ Nicolas VICAIRE \\ Mohammed TAOUIS \\ Laboratoire de Neuroendocrinologie \\ Moléculaire de la Prise Alimentaire, \\ Centre de Neurosciences Paris Sud \\ (CNPS), \\ UMR 8195, \\ CNRS, \\ Université Paris Sud, \\ Faculté des Sciences d'Orsay, \\ F-91405 Orsay, \\ France \\ <claire-marie.vacher@u-psud.fr>
}

\begin{abstract}
Cytokines play an important role in energy-balance regulation. Notably leptin, an adipocyte-secreted cytokine, regulates the activity of hypothalamic neurons that are involved in the modulation of appetite. Leptin decreases appetite and stimulates weight loss in rodents. Unfortunately, numerous forms of obesity in humans seem to be resistant to leptin action. The ciliary neurotrophic factor (CNTF) is a neurocytokine that belongs to the same family as leptin and that was originally characterized as a neurotrophic factor that promotes the survival of a broad spectrum of neuronal cell types and that enhances neurogenesis in adult rodents. It presents the advantage of stimulating weight loss in humans, despite the leptin resistance. Moreover, the weight loss persists several weeks after the cessation of treatment. Hence, CNTF has been considered as a promising therapeutic tool for the treatment of obesity and has prompted intense research aimed at identifying the cellular and molecular mechanisms underlying its potent anorexigenic properties. It has been found that CNTF shares signaling pathways with leptin and is expressed in the arcuate nucleus (ARC), a key hypothalamic region controlling food intake. Endogenous CNTF may also participate in the control of energy balance. Indeed, its expression in the ARC is inversely correlated to body weight in rats fed a high-sucrose diet. Thus hypothalamic CNTF may act, in some individuals, as a protective factor against weight gain during hypercaloric diet and could account for individual differences in the susceptibility to obesity.
\end{abstract}

Key words: CNTF, hypothalamus, energy homeostasis
Obesity is a chronic, metabolic disease of complex and multiple causes leading to an imbalance between energy intake and output, and to the accumulation of large amounts of body fat. It is caused by inherited as well as acquired factors, including excessive food intake, sedentary lifestyle and unhealthy eating habits. During the past 20 years, obesity among adults has risen significantly with urbanization, economic development and market globalization. According to the World Health Organization (WHO) statements, more than one billion people worldwide are overweight or suffer from obesity, and the number of affected children has more than doubled since 1980 in the USA and Europe. In France, the latest data from Roche show that overweighting and obesity affect, respectively, more than $30 \%$ and $14.5 \%$ of adults (ObEpi-Roche, 2009). But far more worrying are the increasing and acceleration of this problem into developing countries and, based on current trends, it is predicted that the levels of obesity will continue to rise unless action is taken now (McLellan, 2002). The consequences of obesity for adults are well known. Obesity contributes to the development of many diseases, including diabetes, hypertension, dyslipidemia (for instance, high total cholesterol or high levels of triglycerides), stroke, cardiovascular disease, and some cancers (Abelson and Kennedy, 2004). As a result, the obesity epidemic has prompted important efforts to develop safe and potent therapies. However, currently approved drugs for obesity such as appetite suppressants have limited efficacy and act acutely, with patients rapidly regaining weight after the cessation of treatment. The neurocytokine ciliary neurotrophic factor (CNTF) seems to deviate from this paradigm since its administration to rodents or patients maintains lowered body weights several weeks after terminating treatment (Lambert et al., 2001).

CNTF is a 200-amino acid cytokine that belongs to the IL- 6 family. It is expressed in both the peripheral and the central nervous systems by neuronal and glial cells. Originally, CNTF was shown to promote the survival of ciliary ganglion neurons (Barbin et al., 1984; Helfand et al., 1976) and to play a major role in

To cite this article: Vacher CM, Couvreur O, Basire E, Aubourg A, Crépin D, Berthou F, Vicaire N, Taouis M. Brain Ciliary Neurotrophic Factor (CNTF) and hypothalamic control of energy homeostasis. OCL 2011; 18(5): 263-266. doi : 10.1684/ocl.2011.0399 
the adult nervous system's early response to lesions. Today, we know that its spectrum of functions is much broader since it includes the differentiation and/ or survival of a variety of nervous cells such as motor neurons, oligodendrocytes and astrocytes (Hughes et al., 1988; Mayer et al., 1994; Sendtner et al., 1992). In an initial clinical trial designed to test the efficacy of a CNTF analogue (Axokine ${ }^{\mathbb{R}}$, Regeneron Pharmaceuticals, Tarrytown, NY) in the treatment of amyotrophic lateral sclerosis, a degenerative motor neuron disease, some patients suffered a substantial weight loss (Miller et al., 1996a; Miller et al., 1996b). Since then the mechanisms by which CNTF induces weight loss have been deciphered using animal models: CNTF mimics the ability of leptin to reduce food intake and to induce fat loss.

Indeed, similar to leptin, an adipocytesecreted cytokine well known for its role in the long-term homeostasis of body weight, CNTF reduces appetite and body fat by providing a signal of energy intake and energy stores in the body to the arcuate nucleus (ARC) of the hypothalamus, a nucleus involved in hunger control (Markus, 2005). Adjacent to the third ventricle and to the median eminence, the ARC is ideally located to be a putative brain sensor of factors circulating in the blood and the cerebrospinal fluid. Notably, ARC integrates changes in circulating levels of nutrients and hormones such as leptin and insulin to respond to the energy body requirements (Schwartz, 2000). The ARC contains two main neuronal populations that exert contrary effects on energy balance. Neuropeptide Y (NPY)-producing neurons stimulate while pro-opiomelanocortin (POMC)-synthesizing neurons inhibit appetite. In rats, the anorexigenic action of exogenous CNTF has been associated to a decrease in NPY gene expression (Xu et al., 1998) and to an increase in POMC transcription (Ambati et al., 2007). Interestingly, the chronic administration of CNTF causes a decrease in food intake and body weight without inducing a rebound effect at the cessation of treatment, usually observed after a sustained reduction in caloric intake. This effect has been attributed to a resensitization of the ARC to leptin due to a CNTF-induced neurogenesis (Kokoeva et al., 2005).

Efforts to understand the mechanisms of action of CNTF in the nervous system have led to the identification of a threecomponent receptor complex for this cytokine. CNTF first binds to its specific CNTF receptor (CNTFR $\alpha)$, which does not play a direct role in signal transduction (Davis et al., 1993a). CNTFR $\alpha$ exists in two forms, membrane bound and soluble. The glycosyl phosphatidylinositol linkage of CNTFR $\alpha$ to the cell membrane can be cleaved by phospho- lipases releasing CNTFR $\alpha$ to act as a soluble protein (Taga et al., 1989). Then, binding of CNTF to the membranebound or soluble CNTFR $\alpha$ induces heterodimerisation of the " $\beta$ " components of the receptor complex, gp130 and LIF receptor $\beta$ (LIFR $\beta$ ), which trigger intracellular signaling cascades (Davis et al., $1993 b)$. The $\beta$ components of CNTF receptor complex are preassociated in

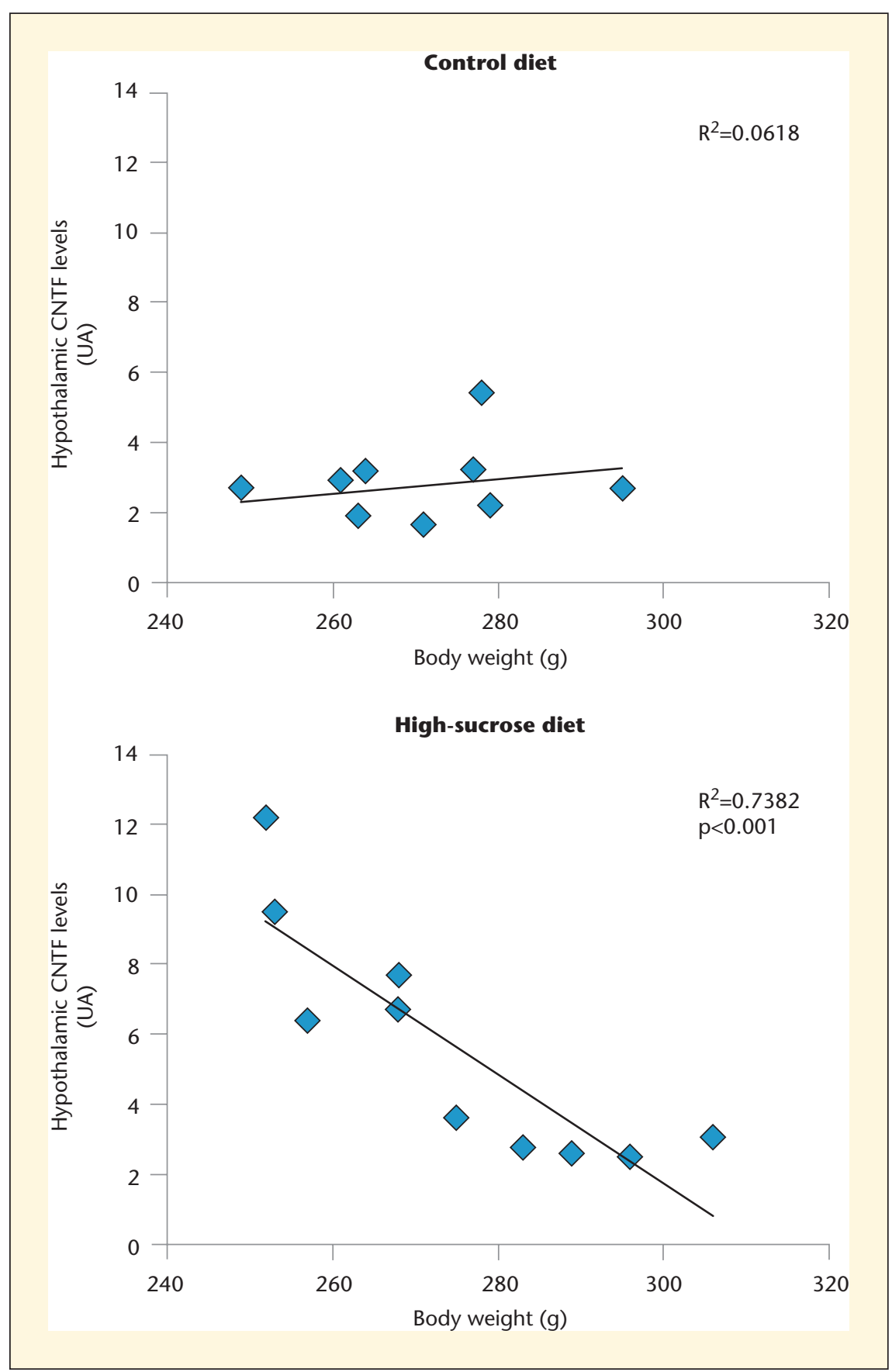

Figure 1. CNTF levels determined in the hypothalamus by Western blot negatively correlates with body weight in high-sucrose diet fed rats but not in control diet fed rats. 
an inactive state with the cytoplasmic Jak/Tyk tyrosine kinases. The $\beta$ component dimerisation initiates the activation of mitogen-activated protein kinase/ extracellular signal-regulated kinase (MAPK/ERK) and Jak/Tyk kinases, which, in turn, phosphorylate the signal transducer and activator of transcription 3 (STAT3). In this condition, phosphoSTAT3 forms a dimer that translocates to the nucleus where it activates the transcription of target genes (Stahl and Yancopoulos, 1993). The activation of this signaling pathway by CNTF is negatively modulated by the suppressor of cytokine signaling (SOCS) family of proteins (Bjorbaek et al., 1999). Thus, in rodents, CNTF shares signaling cascades with leptin in the ARC. More interesting is the fact that CNTF, which signals through leptin-like pathways, has been shown to bypass leptin resistance in diet-induced obesity model, a more representative model of human obesity (Gloaguen et al., 1997; Munzberg et al., 2005). We have shown that leptin but not CNTF is able to induce protein-tyrosine phosphatase-1B (PTP$1 \mathrm{~B})$ expression. In addition, and contrary to leptin, CNTF signaling was not affected by PTP-1B over-expression, suggesting that PTP-1B is a key divergent element between CNTF and leptin signaling pathways. This may at least partially explain the efficacy of CNTF administration to reduce food intake and body weight in leptin resistant state (Benomar et al., 2009).

It is noteworthy that CNTF is highly expressed both in neurons and astrocytes of the hypothalamic nuclei that regulate energy balance, including the POMC anorexigenic neurons located in the ARC. To test the hypothesis of a relationship between the hypothalamic expression of CNTF and the control of energy homeostasis, the influence of a 6-week high-sucrose diet was studied on CNTF levels in the hypothalamus and the ARC in rats (Vacher et al., 2008). The highsucrose diet induces a 2-fold increase in CNTF hypothalamic levels compared to control. Interestingly, while no association is observed between CNTF hypothalamic levels and body weight in control animals, a significant inverse correlation appears in rats fed the highsucrose diet (figure 1). Indeed, in these conditions, animals with lower body weight exhibit higher amounts of CNTF in the hypothalamus. The variations in protein contents parallel those of mRNA levels. Moreover, the increase in CNTF expression is specific to the ARC, as evidenced by an immunohistochemical analysis. Thus, CNTF may be considered as an endogenous modulator of energy homeostasis in the ARC that possibly contributes to the protection of some individuals against diet induced weight gain. CNTF could account for individual differences in the susceptibility to obesity. Genetic polymorphisms studies corroborate the involvement of endogenous CNTF in the control of body weight. Indeed, it has been found that a null mutation in CNTF gene is associated with a significant increase in body mass in humans (Heidema et al., 2010; O'Dell et al., 2002), and that variants in CNTF or CNTFR $\alpha$ gene in humans are associated to lower age at onset of eating disorders (Gratacos et al., 2010).

The anorexigenic properties of exogenous and endogenous CNTF have conferred to this cytokine a promising therapeutic potential in the treatment of obesity. However, the comprehension of the physiological significance of neural CNTF action is still incomplete because CNTF lacks a signal peptide (Sendtner et al., 1994), and thus may not be secreted by the classical exocytosis pathways. We have previously shown that CNTF distribution shares similarities with that of its receptor subunits in the rat ARC. Indeed, a majority of neurons and astrocytes express both CNTF and CNTFR $\alpha$, and both $\beta$ components of the receptor are ubiquitous in the rat ARC (figure 2) (Vacher et al., 2008). Thus, as previously envisaged in cell culture (Monville et al., 2002), a direct intracellular action may constitute a plausible mechanism of CNTF action. The involvement of such a process in the protective action of endogenous CNTF against dietinduced weight gain deserves further investigation. Nevertheless, these data could influence future drug discovery
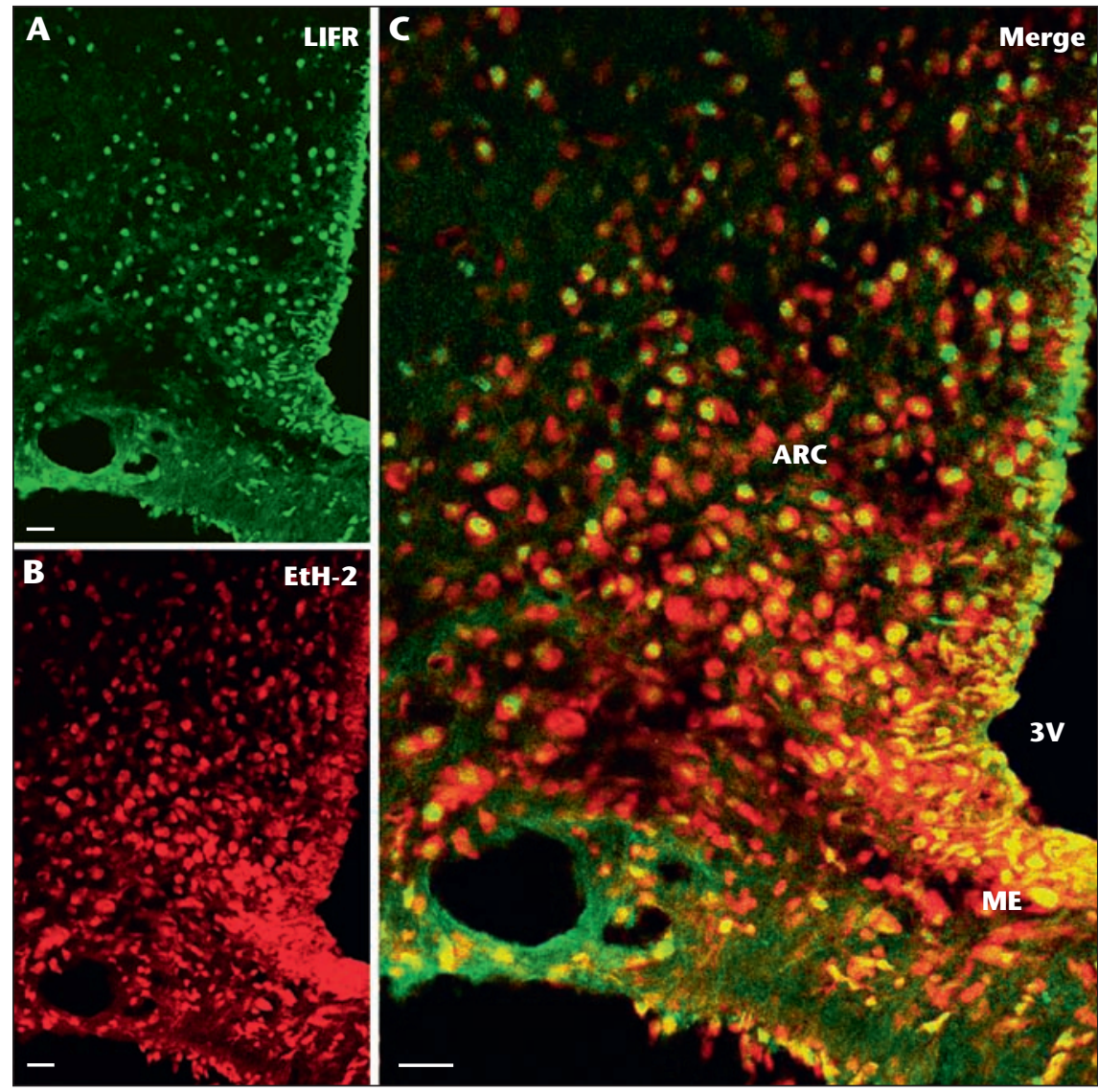

Figure 2. Immunohistochemical detection of LIFR (green) in the rat arcuate nucleus (counterstaining with ethidium homodimer-2 in red). Confocal Z-stacks of three $0.5 \mu \mathrm{m}$-thick focal planes. 3V, third ventricle; ARC, arcuate nucleus; EtH-2, ethidium homodimer-2; LIFR, LIF receptor; $M E$, median eminence. Scale bars $=50 \mu \mathrm{m}$. 
efforts for the development of new therapeutic targets against obesity.

\section{REFERENCES}

Abelson P, Kennedy D. The obesity epidemic. Science 2004; 304: 1413.

Ambati S, Duan J, Duff E, et al. Gene expression in arcuate nucleus-median eminence of rats treated with leptin or ciliary neurotrophic factor. Biofactors 2007; 31: 133-44.

Barbin G, Manthorpe M, Varon S. Purification of the chick eye ciliary neuronotrophic factor. I Neurochem 1984; 43: 1468-78.

Benomar Y, Berthou F, Vacher CM, et al. Leptin But Not Ciliary Neurotrophic Factor (CNTF) Induces Phosphotyrosine Phosphatase-1B Expression in Human Neuronal Cells (SH-SY5Y): Putative Explanation of CNTF Efficacy in Leptin-Resistant State. Endocrinology 2009; 150: 1182-91.

Bjorbaek C, Elmquist JK, El-Haschimi, K, et al. Activation of SOCS-3 messenger ribonucleic acid in the hypothalamus by ciliary neurotrophic factor. Endocrinology 1999; 140: 2035-43.

Davis S, Aldrich TH, Ip NY, et al. Released form of CNTF receptor alpha component as a soluble mediator of CNTF responses. Science 1993a; 259: 1736-9.

Davis S, Aldrich TH, Stahl N, et al. LIFR beta and gp130 as heterodimerizing signal transducers of the tripartite CNTF receptor. Science 1993b; 260: 1805-8.

Gloaguen I, Costa P, Demartis A, et al. Ciliary neurotrophic factor corrects obesity and diabetes associated with leptin deficiency and resistance. Proc Natl Acad Sci U S A 1997; 94: 6456-61.

Gratacos M, Escaramis G, Bustamante M, et al. Role of the neurotrophin network in eating disorders' subphenotypes: Body mass index and age at onset of the disease. J Psychiatr Res. 2010.

Heidema AG, Wang P, van Rossum CT, et al. Sex-specific effects of CNTF, IL6 and UCP2 polymorphisms on weight gain. Physiol Behav 2010; 99: 1-7.

Helfand SL, Smith GA, Wessells NK. Survival and development in culture of dissociated parasympathetic neurons from ciliary ganglia. Dev Biol 1976; 50: 541-7.

Hughes SM, Lillien LE, Raff MC, Rohrer H, Sendtner M. Ciliary neurotrophic factor induces type- 2 astrocyte differentiation in culture. Nature 1988; 335: 70-3.

Kokoeva MV, Yin H, Flier JS. Neurogenesis in the hypothalamus of adult mice: potential role in energy balance. Science 2005; 310: 679-83.

Lambert PD, Anderson KD, Sleeman MW, et al. Ciliary neurotrophic factor activates leptin-like pathways and reduces body fat, without cachexia or rebound weight gain, even in leptin-resistant obesity. Proc Natl Acad Sci U S A 2001; 98: 4652-7.

Markus A. Neurobiology of obesity. Nat Neurosci 2005; 8, 551.

Mayer M, Bhakoo K, Noble M. Ciliary neurotrophic factor and leukemia inhibitory factor promote the generation, maturation and survival of oligodendrocytes in vitro. Development 1994; 120: 143-53.

McLellan F. Obesity rising to alarming levels around the world. Lancet 2002; 359: 1412.

Miller RG, Bryan WW, Dietz MA, et al. Toxicity and tolerability of recombinant human ciliary neurotrophic factor in patients with amyotrophic lateral sclerosis. Neurology 1996a; 47: 1329-31.

Miller RG, Petajan JH, Bryan WW, et al. A placebo-controlled trial of recombinant human ciliary neurotrophic (rhCNTF) factor in amyotrophic lateral sclerosis. rhCNTF ALS Study Group. Ann Neurol 1996b; 39: 256-60.

Monville C, Fages C, Feyens AM, et al. Astroglial expression of the P-glycoprotein is controlled by intracellular CNTF. BMC Cell Biol 2002; 3: 20.

Munzberg $\mathrm{H}$, Bjornholm M, Bates SH, Myers MG Jr. Leptin receptor action and mechanisms of leptin resistance. Cell Mol Life Sci 2005; 62: 642-52.

O'Dell SD, Syddall HE, Sayer AA, et al. Null mutation in human ciliary neurotrophic factor gene confers higher body mass index in males. Eur J Hum Genet 2002; 10: 749-52.

Schwartz MW. Biomedicine. Staying slim with insulin in mind. Science 2000; 289: 2066-7.

Sendtner M, Carroll P, Holtmann B, Hughes $\mathrm{RA}$, Thoenen $\mathrm{H}$. Ciliary neurotrophic factor. J Neurobiol 1994; 25: 1436-53.

Sendtner M, Schmalbruch $H$, Stockli KA, Carroll P, Kreutzberg GW, Thoenen H. Ciliary neurotrophic factor prevents degeneration of motor neurons in mouse mutant progressive motor neuronopathy. Nature 1992; 358: 502-4.

Stahl N, Yancopoulos GD. The alphas, betas, and kinases of cytokine receptor complexes. Cell 1993; 74: 587-90.

Taga T, Hibi M, Hirata Y, et al. Interleukin-6 triggers the association of its receptor with a possible signal transducer, gp130. Cell 1989; 58: 573-81.

Vacher CM, Crepin D, Aubourg A, et al. A putative physiological role of hypothalamic CNTF in the control of energy homeostasis. FEBS Lett 2008; 582: 3832-8.

Xu B, Dube MG, Kalra PS, et al. Anorectic effects of the cytokine, ciliary neurotropic factor, are mediated by hypothalamic neuropeptide Y: comparison with leptin. Endocrinology 1998; 139: 466-73. 